\title{
Estratégia\&Negócios
}

ISSN 1984-3372

http://www.portaldeperiodicos.unisul.br/index.php/EeN/

\section{FATORES POTENCIALIZADORES E RESTRITIVOS À IMPLANTAÇÃO DO BALANCED SCORECARD: UM ESTUDO EM UMA EMPRESA DE CONSTRUÇÃO CIVIL DO CEARÁ}

\section{POTENTIALIZING AND RESTRICTIVE FACTORS IN THE IMPLEMENTATION OF THE BALANCED SCORECARD: A STUDY IN A CONSTRUCTION FIRM IN THE STATE OF CEARÁ}

\section{Roberta Catrib de Azevedo Lemos}

Universidade Federal do Ceará

E-mail: robertacatrib@portofreire.com.br

\section{Maria Naiula Monteiro Pessoa}

Universidade Federal do Ceará

E-mail: naiula@ufc.br

\section{Sandra Maia dos Santos}

Universidade Federal do Ceará

E-mail: smsantos@ufc.br

\section{Augusto Cézar de Aquino Cabrak}

Universidade Federal do Ceará

E-mail: cabral@ufc.br

\section{Debora Cardoso do Nascimento}

Universidade Federal do Ceará

E-mail: deboracardoso33@hotmail.com

Recebido em 28/07/2011. Aprovado em 18/10/2011. Disponibilizado em 03/12/2011.

Avaliado pelo Sistema double blind review

R. eletr. estrat. neg., Florianópolis, v.4, n.2, p.3-29, jun./dez. 2011

http://portaldeperiodicos.unisul.br/index.php/EeN/index
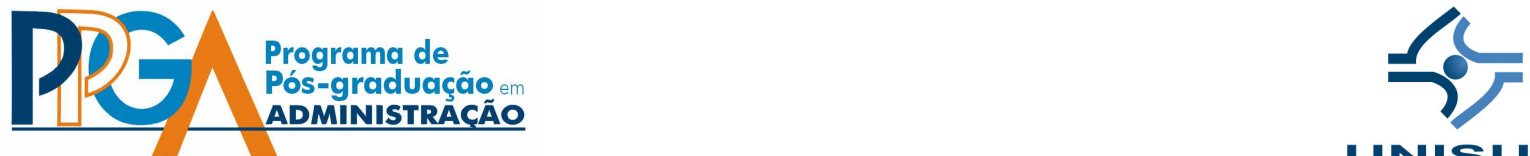

UNISUL

CCopyright 2008 UNISUL-PPGA/Estratégia e Negócios. Todos os direitos reservados. Permitida citação parcial, desde que identificada a fonte. Proibida a reprodução total. Em caso de dúvidas, consulte o editor:

ademar.unisul@gmail.com; (48) 3229-1932. 
Roberta Catrib de Azevedo Lemos - Maria Naiula Monteiro Pessoa - Sandra Maia dos Santos - Augusto Cézar de Aquino Cabrak Debora Cardoso do Nascimento

\section{RESUMO}

O Balanced Scorecard (BSC), mais conhecido como um método de tradução da missão e estratégia da empresa em um conjunto abrangente de medidas de desempenho tem sido utilizado para a gestão estratégica das organizações, atuando como um sistema de medição que, além dos objetivos financeiros, busca incluir características de desempenho. Nesta pesquisa, realizada mediante um estudo de caso, tem-se como objetivo identificar os principais fatores potencializadores e restritivos à implantação do BSC em uma empresa de construção civil do estado do Ceará, a Porto Freire Engenharia. Na pesquisa documental, considerando-se os relatórios gerencias do período de 2003 a 2009, constatou-se que a empresa realizou o processo necessário para a implantação do BSC para a gestão de sua estratégia. A coleta de dados de campo foi realizada mediante um questionário e um roteiro de entrevista, aplicados, respectivamente, aos colaboradores e gestores que já eram integrantes da empresa no momento em que o BSC foi implantado. Quanto aos dados do questionário, os resultados indicam que, do conjunto dos vinte fatores considerados, apenas três foram apontados como potencializadores à implantação do BSC: suporte técnico de consultorias, balanceamento das quatro perspectivas e preservação da identidade corporativa. Em relação aos dados das entrevistas, as práticas de planejamento, as consultorias e a mensuração da estratégia foram apontadas como fatores potencializadores. A cultura organizacional, o processo de sensibilização dos colaborares e a perspectiva e alcance dos resultados foram considerados fatores restritivos.

Palavras-chave: Estratégia. Balanced scorecard. Construção civil.

\section{INTRODUÇÃO}

A busca de uma vantagem competitiva sustentável é uma realidade no dia a dia das organizações. No entanto, esta busca não é algo simples e nem todas as empresas alcançam bons 
Roberta Catrib de Azevedo Lemos - Maria Naiula Monteiro Pessoa - Sandra Maia dos Santos - Augusto Cézar de Aquino Cabrak Debora Cardoso do Nascimento

resultados. Ao mesmo tempo, muitas organizações incorrem em vários erros antes de alcançar o acerto tão desejado.

Na construção civil, a realidade não poderia ser diferente. As empresas do setor têm procurado se manter com uma postura muito proativa no que diz respeito a esta busca constante pela competitividade, inclusive muito focadas em inovações de produto e processos. Oliveira e Freitas (2001) percebem a indústria da construção civil como detentora de certas especificidades que não podem ser desconsideradas em nenhuma análise gerada. Nesse sentido, este estudo, no intuito de reduzir os riscos de um possível e indesejado insucesso, quando da implantação do Balanced Scorecard (BSC)em uma empresa de construção civil, partiu do seguinte problema de pesquisa: Quais os principais fatores potencializadores e restritivos à implantação do BSC em uma empresa de construção civil do estado do Ceará?

Em consonância com esse problema, o trabalho teve como objetivo identificar os principais fatores potencializadores e restritivos à implantação do Balanced Scorecard em uma empresa de construção civil do estado do Ceará, Porto Freire Engenharia, motivado pelo desempenho alcançado por essa empresa. Além desta introdução, o trabalho está estruturado em outros quatro segmentos, sendo o primeiro o do referencial teórico, o segundo a metodologia, o terceiro a análise dos dados e, por fim, a conclusão.

\section{BALANCED SCORECARD}

Com os novos padrões de concorrência, os executivos precisam de indicadores sobre diversos aspectos do ambiente e desempenho organizacional. É nesse contexto que está inserido o Balanced Scorecard. Mais conhecido como um método de tradução da missão e estratégia da empresa em um conjunto abrangente de medidas de desempenho, o BSC atua como um sistema de gestão estratégica alicerçado em quatro perspectivas: financeira, dos clientes, dos processos internos da empresa e do aprendizado e crescimento. No entanto, essas perspectivas são apenas uma proposta do modelo original, a organização pode considerar quaisquer outras perspectivas que considere adequadas (KAPLAN; NORTON, 1997). 
Roberta Catrib de Azevedo Lemos - Maria Naiula Monteiro Pessoa - Sandra Maia dos Santos - Augusto Cézar de Aquino Cabrak Debora Cardoso do Nascimento

Para Campos (1998), o modelo recebeu essa denominação devido ao fato de uma organização ser considerada como percorrendo o caminho do sucesso se esses quatro conjuntos de indicadores estiverem balanceados e, para isso, eles precisam ser aplicados com graus de importância relativa, mas equitativa. Apenas dessa maneira, a organização poderá ser capaz de alcançar um desenvolvimento real e equilibrado. Como o modelo apresenta uma configuração bastante simplificada, ele permite aos gerentes uma visão rápida e completa da situação da organização. No entanto, a empresa precisa partir de uma estratégia adequada às suas atividades e negócios, visto que á proposta do BSC é a de que seja complementar ao plano estratégico, permitindo acompanhar os progressos alcançados ao longo do tempo.

Mackenzie e Shillig (1998) realizaram uma comparação entre o Balanced Scorecard e os modelos tradicionais, apoiados por indicadores financeiros. O BSC apresenta vantagens em relação aos demais, visto que o foco da gestão está direcionado à estratégia e à visão e não mais ao controle. Além disso, a proposta de integração das quatro perspectivas pode ser vista como vantajosa, pois diversas empresas já trabalhavam com perspectivas financeiras e não-financeiras, mas ainda não se tinha concebido estas perspectivas integradas. Dessa maneira, a organização pode ser capaz de alcançar os seus objetivos estratégicos, mantendo o foco nas peculiaridades do seu negócio, ao invés de se lançar na procura de controles desnecessários. Com essa integração, pode-se executar a estratégia de maneira muito mais eficiente.

Olve, Roy e Wetter (2001), por sua vez, apontaram algumas críticas ao controle do gerenciamento tradicional, com foco somente em aspectos financeiros: o fornecimento de informações distorcidas para a tomada de decisão; a negligência em relação às exigências e estratégias da organização; o estímulo dado ao pensamento de curto prazo e subotimização; a posição secundária quanto às exigências do relatório financeiro; o oferecimento de informações distorcidas para a destinação do custo e controle dos investimentos; o fornecimento de informação abstrata para os empregados; e a pouca atenção dada ao ambiente do negócio. Afora essas questões, um importante diferencial, citado por Kaplan e Norton (1992), é que o BSC coloca a estratégia no centro e não o controle. Os modelos tradicionais determinam e monitoram as ações a serem seguidas, tentando, assim, controlar comportamentos. Já o scorecard tem como foco a determinação das metas, partindo do princípio que as pessoas tomarão quaisquer ações e 
Roberta Catrib de Azevedo Lemos - Maria Naiula Monteiro Pessoa - Sandra Maia dos Santos - Augusto Cézar de Aquino Cabrak Debora Cardoso do Nascimento

comportamentos para realizá-las. O foco do controle é completamente direcionado para a execução das metas.

\subsection{EXPERIÊNCIAS NO USO DO BALANCED SCORECARD EM ORGANIZAÇÕES}

Em muitos casos, o Balanced Scorecard trouxe benefícios. Como Kaplan e Norton (1997) evidenciam, diversas empresas alcançaram níveis de satisfação muito elevados quanto à execução de suas estratégias. No entanto, também se encontra na literatura organizações que não obtiveram ganhos reais, ou não foram capazes de perceber os ganhos obtidos.

Bessa (2005) estudou a implantação do scorecard no SENAI do Ceará. A pesquisa evidenciou os elementos potencializadores e restritores. Os elementos potencializadores foram: 0 suporte e decisão das lideranças no âmbito nacional; a adesão das lideranças no âmbito regional e o suporte técnico e profissional da consultoria externa. Como elementos restritivos destacaramse: o grau de autonomia dos departamentos regionais, dificultando a padronização de procedimentos; a necessidade de se pensar e agir estrategicamente em toda a organização,e a cobrança por resultados de curto prazo.

Galas e Ponte (2005) empreenderam uma pesquisa com diversas empresas com o objetivo de analisar o BSC, de modo a identificar fatores que contribuem para o alinhamento organizacional. Os resultados mostraram que algumas dessas empresas ainda não utilizam todo o potencial do BSC, visto que nem todas a utilizam como um sistema de gestão estratégica. Outro resultado foi que a integração das diversas perspectivas do BSC em uma relação de causa e efeito contribuiu para o alinhamento organizacional, pois levou a organização a orientar-se não só a favor de fatores financeiros.

Cavalcante Neto (2004) estudou duas empresas de construção civil e se propôs a avaliar o processo de implantação do modelo do scorecard, além das barreiras enfrentadas pelas organizações. O resultado de uma das empresas estudadas foi que não houve barreira alguma relacionada à presença de liderança, conflitos de interesses, estrutura e adequação do processo e incentivos. No entanto, existiram barreiras quanto à qualidade da estratégia e domínio dos conhecimentos sobre estratégia. No caso da outra empresa avaliada, foram suplantadas barreiras relativas à qualidade da estratégia; pouco domínio sobre conhecimentos de estratégia; ausência de liderança; conflitos de interesses; insuficiência de recursos e inadequação do processo e 
Roberta Catrib de Azevedo Lemos - Maria Naiula Monteiro Pessoa - Sandra Maia dos Santos - Augusto Cézar de Aquino Cabrak Debora Cardoso do Nascimento

estrutura. As barreiras não suplantadas foram: desconhecimento da estratégia da organização; conflito entre as ações estratégicas e o dia a dia; inexistência do monitoramento da execução da estratégia e a ausência de incentivos.

Prieto et al. (2006) mostram os fatores críticos de sucesso na implementação do BSC, bem como o processo de mudança e as técnicas envolvidas na implementação. Um dos problemas mais comuns diz respeito ao envolvimento do gerente de nível sênior, no momento em que os executivos delegam a estratégia de implantação ao nível hierárquico intermediário, os quais podem não estar preparados para compreender a estratégia e o projeto do scorecard. 0 comprometimento da gerência é tão importante para o sucesso quanto conhecimento e sabedoria, tendo como função a divisão das atividades e responsabilidades. Outro fator é que algumas empresas não conseguem traduzir a estratégia nos objetivos do BSC, gerando um processo completamente distorcido de medições e análises.

Acrescente-se a esses casos citados, o modelo implementado pelo Banco do Brasil, atualmente remodelado. Essa pesquisa constatou algumas fragilidades em relação à aplicação do BSC: os fatores são avaliados unilateralmente e descendentemente, além de serem utilizados como instrumentos de pressão; ausência de acompanhamento e feedback por parte dos gestores, sendo esse fator motivo de muitas críticas para scorecards; processo pouco participativo e pouco democrático, também considerado no scorecard como um fator restritivo ao processo e alguns conflitos de poder na organização. O Banco do Brasil teve, então, que reformular esse modelo de gestão e criou um grupo de trabalho multidisciplinar para esta atividade, com foco nas fragilidades do modelo. Foram realizados testes do instrumento de gestão e obtiveram-se resultados muito positivos, mas a implantação final está sendo tratada com cautela para não se cometer os mesmos erros (BRANDÃO et al. 2008).

Campos (1998) afirma a importância de se reavaliar a cultura organizacional antes da implantação do BSC. Prado (2009) acrescenta que o BSC abrange toda a organização e não uma minoria, de maneira que seria um grande erro implantá-lo sem que seja revisto o formato cultural da empresa. Além da cultura, a comunicação deve ser reavaliada, pois o BSC precisa ser amplamente divulgado, até mesmo quando os resultados não sejam favoráveis. Outros pontos importantes são organização, pontualidade, identidade e tecnologia. Herrero Filho (2005) ressalta a relevância da comunicação da estratégia e do alinhamento estratégico. 
Roberta Catrib de Azevedo Lemos - Maria Naiula Monteiro Pessoa - Sandra Maia dos Santos - Augusto Cézar de Aquino Cabrak Debora Cardoso do Nascimento

O Quadro 1 sintetiza os pontos críticos na implantação do BSC, conforme a literatura.

Quadro 1: Pontos críticos à implantação do BSC apresentados na literatura.

\begin{tabular}{|c|c|}
\hline Autores Pesquisados & Fatores Restritivos ou Potencializadores \\
\hline Kaplan e Norton (2000) / Campos (1998) / Prado (2009) & Cultura Organizacional \\
\hline \multirow{3}{*}{ Kaplan e Norton (1997) / Brandão et al. (2008) } & Provisionamento de recursos financeiros \\
\hline & Fornecimento de feedback \\
\hline & Comprometimento dos colaboradores \\
\hline \multirow{2}{*}{ Herrero Filho (2005) / Bessa ( 2005) / Galas e Ponte (2005) } & Comunicação da estratégica \\
\hline & Alinhamento estratégico \\
\hline \multirow{2}{*}{ Kaplan e Norton (2000) / Olve, Roy e Wetter (2001) } & Comprometimento da altadireção \\
\hline & Planejamento \\
\hline \multirow{3}{*}{ Bessa (2005) / Prieto et al (2006) } & Adesão dos gestores \\
\hline & Suporte de consultorias \\
\hline & Presença do pensamento estratégico \\
\hline $\begin{array}{l}\text { Kaplan e Norton (1997) / Galas e Ponte (2005) / Prieto et al } \\
\text { (2006) }\end{array}$ & Balanceamento das perspectivas \\
\hline \multirow{3}{*}{ Cavalcante Neto (2004) / Prieto et al. (2006) } & Estrutura da organização \\
\hline & Mapeamento dos processos \\
\hline & Conflitos de interesses \\
\hline \multirow{2}{*}{ Kaplan e Norton (1997) / Cavalcante Neto (2004) } & Qualidade da estratégia \\
\hline & Incentivos financeiros \\
\hline \multirow{4}{*}{ Prado (2009) } & Organização \\
\hline & Cumprimento do cronograma \\
\hline & Identidade organizacional \\
\hline & Aspectos tecnológicos \\
\hline
\end{tabular}

Fonte: elaborado pelos autores (2010).

No levantamento feito, percebe-se que alguns pontos foram citados por quase todos os autores pesquisados. Outros pontos foram menos mencionados. No entanto, no estudo de caso, optou-se por analisar todos os pontos críticos apresentados.

\section{PERCURSO METODOLÓGICO}


Roberta Catrib de Azevedo Lemos - Maria Naiula Monteiro Pessoa - Sandra Maia dos Santos - Augusto Cézar de Aquino Cabrak Debora Cardoso do Nascimento

Este trabalho consistiu em um estudo transversal, analítico, sobre os fatores restritivos e potencializadores para a implantação do Balanced Scorecard, em uma empresa de construção civil. Trata-se de um estudo exploratório descritivo. Quanto aos procedimentos, a pesquisa foi bibliográfica, documental e estudo de caso.

Trata-se de um estudo de caso único (YIN, 2005), pois se apresenta como um estudo intenso e profundo acerca de um assunto, tendo como foco uma organização. A pesquisa foi realizada em uma empresa de construção civil de grande porte do estado do Ceará, tomando-se como parâmetro o artigo 17 D da Lei no 10.165, de 2000, que designa como classificadas nessa categoria as empresas que tiverem receita bruta anual superior a doze milhões de reais. A empresa foi a Porto Freire Engenharia. A Porto Freire é uma empresa que atualmente conta com cento e sessenta e dois funcionários no seu quadro funcional, e atua no mercado cearense de construção civil desde 1984 de uma maneira muito peculiar. Ela trabalha com construção a preço de custo e desenvolveu seu próprio modelo de financiamento, o sistema Prevcon. Este modelo foi desenvolvido com o intuito de apresentar um sistema de compras programadas, em que o cliente realiza um planejamento e programa o seu futuro (PORTO FREIRE, 2010).

O estudo aconteceu em duas etapas, sendo a primeira a aplicação de um questionário, que após um pré-teste, foi direcionado a todos os colaboradores que já eram integrantes da organização no momento em que o BSC foi implantado. Este instrumento foi aplicado mediante envio on-line, por meio do correio eletrônico da empresa, acompanhado de carta dos pesquisadores com os objetivos do estudo e a contribuição que o colaborador poderia dar ao desenvolvimento da pesquisa ao devolver, também via e-mail, o questionário respondido, no prazo solicitado. Foi informado aos respondentes que os resultados seriam analisados em sua totalidade, preservando-se, assim, o sigilo. Participaram da pesquisa vinte e um colaboradores.

A segunda etapa constou de uma entrevista semiestruturada aplicada apenas à alta administração. As entrevistas ocorreram em horário e local acordados antecipadamente e foram gravadas com autorização prévia dos entrevistados, os quais foram também informados sobre a preservação de suas identidades e a análise dos resultados de forma global.

O questionário era formado por três blocos de questões. O primeiro bloco visava o levantamento do perfil do respondente, tendo como tópicos: sexo, idade, escolaridade, tempo de trabalho e área na empresa, e participação na implantação do BSC.O segundo bloco, composto por 
Roberta Catrib de Azevedo Lemos - Maria Naiula Monteiro Pessoa - Sandra Maia dos Santos - Augusto Cézar de Aquino Cabrak Debora Cardoso do Nascimento

vinte afirmativas acerca da ocorrência de atividades/práticas da empresa ao longo do processo de implementação do BSC, sumarizados no Quadro 2, trazia uma escala de valoração, em que o respondente assinalou o grau de concordância (muito em desacordo; parcialmente em desacordo; parcialmente em acordo; totalmente em acordo) e, também, o grau de relevância (nada importante; pouco importante; importante; muito importante) atribuídos a cada uma destas questões. Estas atividades/práticas foram, então, identificadas como sendo fatores potencializadores ou restritivos.

$\mathrm{Na}$ escala de valoração, o colaborador fornecia o grau de realização de cada afirmação na empresa, representado por uma escala de 1 a 4, sendo 1 muito em desacordo, 2 parcialmente em desacordo, 3 parcialmente em acordo e 4 totalmente em acordo. Além disso, também era fornecido o grau de importância de cada fator para esta empresa, representado por uma escala de $A$ a $D$, sendo A nada importante, B pouco importante, C importante e D muito importante.

Quadro 2 - Fatores potencializadores ou restritivos na implementação do BSC.

\begin{tabular}{|c|c|}
\hline $\begin{array}{l}\text { 1. Participação da alta direção na sensibilização dos } \\
\text { colaboradores. }\end{array}$ & 11. Tomada de decisões em tempo hábil. \\
\hline 2. Comprometimento dos colaboradores. & 12. Suporte técnico de consultorias. \\
\hline 3. Identificação prévia das mudanças necessárias. & $\begin{array}{l}\text { 13. Pensamento estratégico nas tomadas de } \\
\text { decisões. }\end{array}$ \\
\hline 4. Comunicação do processo em todas as etapas. & 14. Resolução prévia de conflitos de interesses. \\
\hline 5. Motivação dos colaboradores durante o processo. & 15. Disponibilização dos recursos necessários. \\
\hline $\begin{array}{l}\text { 6. Promoção de diálogos transparentes e sem } \\
\text { restrições. }\end{array}$ & 16. Consideração dos aspectos culturais no processo. \\
\hline $\begin{array}{l}\text { 7. Escolha de estratégia compatível com objetivos da } \\
\text { empresa. }\end{array}$ & 17. Balanceamento das quatro perspectivas do BSC. \\
\hline $\begin{array}{l}\text { 8. Mapeamento dos processos e padronização das } \\
\text { atividades da empresa. }\end{array}$ & 18. Preservação da identidade corporativa. \\
\hline $\begin{array}{l}\text { 9. Fornecimento de feedback, por parte dos } \\
\text { gestores, em todas as fases. }\end{array}$ & $\begin{array}{l}\text { 19. Comportamento ativo dos envolvidos na } \\
\text { resolução dos problemas. }\end{array}$ \\
\hline $\begin{array}{l}\text { 10. Organização interna da empresa para a } \\
\text { implantação. }\end{array}$ & 20. Obediência ao cronograma estabelecido. \\
\hline
\end{tabular}

Fonte: elaborado pelos autores (2010).

O terceiro bloco do questionário, composto por dezessete tópicos, apresentados no Quadro 3, acerca de características da empresa em estudo, trazia uma escala do tipo Likert, com opções de 1 a 7, de maneira que os entrevistados deviam qualificar cada um desses aspectos a partir da escala de um a sete, na qual o valor um representava a menor qualificação ou fatores restritivos e o valor sete a maior qualificação ou fatores potencializadores à implantação do BSC 
Roberta Catrib de Azevedo Lemos - Maria Naiula Monteiro Pessoa - Sandra Maia dos Santos - Augusto Cézar de Aquino Cabrak Debora Cardoso do Nascimento

nesta empresa. Sendo assim, o respondente assinalou sua visão quanto ao grau de contribuição negativa (quanto mais próximo de 1) ou positiva (quanto mais próximo de 7) de cada uma das características apresentadas para o processo de implementação do BSC.

Quadro 3 - Características da empresa em estudo.

\begin{tabular}{|ll|l|l|}
\hline 1. & Porte da empresa & 7. Sistema de informação & 13. Nível de planejamento \\
\hline 2. & Número de colaboradores & 8. Disponibilidade financeira & 14. Nível de organização \\
\hline $3 . \quad$ Modelo de gestão & 9. Conhecimento do BSC & 15. Experiência no ramo \\
\hline $4 . \quad$ Ramo de negócio dos & $\begin{array}{l}\text { 10. Estrutura hierárquica } \\
\text { informações }\end{array}$ & 16. Característica inovadora \\
\hline $\begin{array}{l}5 . \quad \text { Escolaridade de } \\
\text { colaboradores }\end{array}$ & Mecanismos & 17. Volume de obras \\
\hline $\begin{array}{l}\text { 12. Ocorrência de mudanças } \\
\text { informação }\end{array}$ & ---------------- \\
\hline
\end{tabular}

Fonte: elaborado pelos autores (2010).

Os dados do questionário receberam um tratamento estatístico através do SPSS. Fezse uso da estatística descritiva, no caso específico, média e frequência relativa das respostas obtidas, para consolidação das informações levantadas. A partir dos resultados, foram analisados o grau de relevância dos fatores na implantação de um BSC, bem como o efetivo grau de realização destes fatores no BSC da empresa, tendo sido consideradas relevantes as respostas nas categorias importante ou muito importante e, no tocante ao grau de realização, as respostas na categoria totalmente em acordo.

Para a análise das entrevistas, as falas foram estruturadas de forma organizada sem que se perdesse a noção do todo, porém também estavam sujeitas a uma análise mais completa, baseada na subjetividade de cada sujeito, em que se procura perceber o significado do discurso, realizando-se, assim, um trabalho bem mais aprofundado e fidedigno. Com base nos dados apreendidos nessa fase da coleta, realizou-se um confronto destes, considerando o que foi identificado nas respostas às questões da entrevista. Tanto os itens dos questionários como os tópicos das entrevistas versavam sobre os mesmos aspectos da implantação da ferramenta BSC. Utilizou-se para organização dos dados a técnica de categorização das falas dos sujeitos.

A partir da análise de conteúdo (BARDIN, 2008), definiram-se as categorias de análise das falas expressas nas entrevistas realizadas com a alta direção, compreendendo três diretores. Tomando como base as questões fundamentais norteadoras da implantação do BSC em uma empresa do ramo de construção civil, foram identificadas as manifestações dos sujeitos que 
Roberta Catrib de Azevedo Lemos - Maria Naiula Monteiro Pessoa - Sandra Maia dos Santos - Augusto Cézar de Aquino Cabrak Debora Cardoso do Nascimento

constituem o segmento ativo de gestão na referida organização. Organizaram-se os dados qualitativos em seis categorias: a) Dependência do planejamento para o sucesso da execução da estratégia; b) Objetivação da estratégia da organização; c) Aparato teórico e técnico da consultoria na implantação; d) Sensibilização dos envolvidos para a utilização do BSC como instrumento de gestão; e) Processo de construção de cultura organizacional direcionada ao trabalho disciplinar e f) Perspectiva de alcance dos resultados esperados.

\section{O BALANCED SCORECARD NA PORTO FREIRE ENGENHARIA}

A Porto Freire Engenharia, como atestam seu perfil institucional e seu histórico, caracteriza-se como uma empresa inovadora, focada em modernização de processos produtivos e gerenciais. Adota peculiaridades e estratégias direcionadas ao planejamento, pois foi concebida para construir pelo sistema a preço de custo, em que as obras são autofinanciadas, o que requer planejamento dos clientes e da construtora. Optou-se por analisar a série histórica de relatórios gerenciais de 2003 - 2009, relativos ao planejamento estratégico e ao Balanced Scorecard. Foram analisados os seguintes elementos:

- Mapa estratégico - foi construído de acordo com as relações de causa e efeito e baseia-se nas quatro perspectivas propostas por Kaplan e Norton (1997);

- Missão - definida com foco no propósito da organização, que sempre esteve ligado à prosperidade das pessoas, pois a empresa busca desenvolver grupos de consumidores e fornecedores e não apenas alcançar crescimento próprio;

- Visão - focada na liderança de uma vantajosa cadeia de suprimentos, viabilizando benefícios para os clientes. A empresa mantém uma postura proativa em relação à inovação e aperfeiçoa essa visão a cada planejamento.

- Valores - o planejamento estratégico demonstra a preocupação em inovar, aprender e planejar, dentre outros. Prevalece uma abordagem com foco em valores humanos, no sentido de fortalecer a sua identidade; 
Roberta Catrib de Azevedo Lemos - Maria Naiula Monteiro Pessoa - Sandra Maia dos Santos - Augusto Cézar de Aquino Cabrak Debora Cardoso do Nascimento

- Estratégias - a empresa tem como estratégia buscar a prosperidade por meio do planejamento, sendo essa a sua maior vantagem competitiva. Apresenta uma estratégia em relação a custo, gerindo os recursos de maneira eficiente e eficaz, além de manter sérias parcerias;

- Perspectivas do BSC - a empresa desenvolveu o seu scorecard considerando apenas as quatro perspectivas do modelo original, sem acréscimos ou subtrações. Toda a estratégia foi aderente a esse modelo.

Os documentos analisados demonstraram que a empresa realizou o processo necessário para a implantação do BSC para a gestão de sua estratégia, pois partiu de uma análise SWOT e evoluiu com o planejamento estratégico. Além disso, os documentos comprovam que foi implantada a certificação ISO antes do BSC, de maneira que os processos estavam todos mapeados e padronizados, o que forneceu agilidade e eficiência ao processo.

\subsection{A PERSPECTIVA DOS COLABORADORES SOBRE A IMPLANTAÇÃO DO BSC}

Quanto ao perfil dos respondentes, $67 \%$ são do sexo feminino. Além disso, 5\% possuem ensino médio, $29 \%$ ensino superior e $66 \%$ possuem pós-graduação, de maneira que $95 \%$ dos participantes possuem ensino superior completo. No que concerne à idade, obteve-se uma média de 35 anos. Quanto ao tempo de trabalho, 67\% trabalham na empresa há, no máximo dez anos; $28 \%$ trabalham na empresa de dez a quinze anos e $5 \%$ acima de quinze anos. Todos os colaboradores que participaram da pesquisa já faziam parte de seu quadro funcional quando o BSC foi implantado em 2003. Em relação à participação na implantação, $86 \%$ participaram ativamente e apenas 14\% não participaram. Este alto grau de participação deve ser visto como um fator positivo que gerou o envolvimento de profissionais de diversas áreas: $28 \%$ eram da área técnica, $14 \%$ da área financeira, $14 \%$ do setor de desenvolvimento de produto, $9 \%$ do setor comercial e os $35 \%$ restantes das áreas administrativa, qualidade, contabilidade, relacionamento, comunicação, marketing e tecnologia da informação.

\subsubsection{Fatores potencializadores e restritivos ao BSC na visão dos colaboradores}


Roberta Catrib de Azevedo Lemos - Maria Naiula Monteiro Pessoa - Sandra Maia dos Santos - Augusto Cézar de Aquino Cabrak Debora Cardoso do Nascimento

Neste segmento, são apresentados os resultados da pesquisa em relação aos vinte fatores considerados na implantação do Balanced Scorecard, conforme sumarizado no Quadro 2, na metodologia. Estes dados referem-se ao segundo bloco do questionário.

- Participação da alta direção na sensibilização dos colaboradores

Kaplan e Norton (1997) ressaltam a importância do envolvimento da alta direção na sensibilização dos colaboradores para a implantação do BSC. Embora todos concordem com a importância do envolvimento da alta direção, apenas $48 \%$ concordam que este envolvimento tenha efetivamente acontecido. Dessa maneira, o envolvimento da alta direção, na empresa, portou-se como fator restritivo.

- Comprometimento dos colaboradores durante todo o processo

Kaplan e Norton (1997) apresentam o comprometimento dos colaboradores como um ponto a ser estimulado. Aproximadamente, $95 \%$ dos respondentes julgam este fator importante ou muito importante. Contudo, na empresa apenas $19 \%$ estão totalmente em acordo que este comprometimento tenha acontecido; logo, não se pode dizer que este tenha sido um fator potencializador.

- Identificação prévia das mudanças necessárias

Herrero Filho (2005) afirma que para se implantar um BSC, deve-se identificar as mudanças necessárias. Aproximadamente $90 \%$ dos participantes julgaram a identificação prévia da necessidade de mudança como um fator importante ou muito importante. Contudo, somente 19\% estão totalmente em acordo que tenha havido a efetiva identificação prévia das mudanças na Porto Freire Engenharia. Logo, este é um fator restritivo.

- Comunicação do processo em todas as etapas

Herrero Filho (2005) identificou que diversas empresas que falharam na implantação do BSC associavam esse fracasso a um processo insuficiente de comunicação nas etapas da implantação. Embora todos concordem com a importância deste fator na implantação do BSC, ele foi considerado fator restritivo dado que apenas $14,4 \%$ dos participantes concordaram totalmente que esse processo tenha sido eficiente.

- Incentivo para motivação dos colaboradores 
Roberta Catrib de Azevedo Lemos - Maria Naiula Monteiro Pessoa - Sandra Maia dos Santos - Augusto Cézar de Aquino Cabrak Debora Cardoso do Nascimento

Cavalcante Neto (2004) identificou a relevância de existência de incentivos para motivação dos colaboradores na implantação de um modelo de gestão. A maioria dos respondentes, $81 \%$, considerou este fator importante ou muito importante. Porém, na empresa apenas $14,3 \%$ estavam totalmente em acordo, indicando que os incentivos ou não foram percebidos ou não existiram durante o processo. Logo, este fator foi considerado restritivo.

- Promoção de diálogos transparentes e sem restrições

A grande maioria dos participantes, 97\%, consideram importante ou muito importante a existência de diálogos transparentes e sem restrições, em alinhamento à visão de Kaplan e Norton (1997). Contudo, apenas 14,3\% afirmaram estar totalmente em acordo com a ocorrência deste fator na empresa. Logo, este foi considerado um fator restritivo.

- Escolha de estratégia compatível com objetivo da empresa Kaplan e Norton (1997) salientam a relevância da escolha de uma estratégia alinhada aos objetivos da empresa. A totalidade dos participantes da pesquisa julgou este fator relevante ou muito relevante. Ao mesmo tempo, $42,9 \%$ dos respondentes afirmaram estar totalmente em acordo de que a escolha da estratégia da empresa esteja compatível com o objetivo da Porto Freire. Portanto, este também foi considerado um fator restritivo.

- Mapeamento dos processos e padronização das atividades da empresa

$\mathrm{Na}$ implantação de um BSC, Kaplan e Norton (1997) recomendam que a organização defina uma cadeia de valor completa dos processos internos que tenha início com o processo de inovação, prossiga como os processos de operações e, termine com o serviço pós-venda. Em relação a esse aspecto, 95,2\% dos respondentes consideraram que seja importante ou muito importante o mapeamento dos processos para a potencialização da implantação do BSC. Ao mesmo tempo em que $47,6 \%$ afirmam estar totalmente em acordo que a empresa tenha realizado o efetivo mapeamento; até mesmo porque a empresa possui um sistema de gerenciamento desses documentos, que se fez necessário, devido à implantação da certificação ISO. Não houve $50 \%$ dos participantes completamente de acordo com esse quesito, por isso esse quesito pode ser considerado como restritivo.

- Fornecimento de feedback pelos gestores 
Roberta Catrib de Azevedo Lemos - Maria Naiula Monteiro Pessoa - Sandra Maia dos Santos - Augusto Cézar de Aquino Cabrak Debora Cardoso do Nascimento

O feedback é capaz de estimular adaptações à estratégia, de acordo com a situação que a organização possa vir a enfrentar, tornando-se um ponto importante para a implantação do BSC (KAPLAN; NORTON, 2000). Do total de participantes, $81 \%$ afirmaram que o feedback é importante ou muito importante. No entanto, apenas $14,4 \%$ dos participantes estavam totalmente em acordo quanto ao fornecimento efetivo de feedback pelos gestores no processo de implantação do BSC nesta organização, o que indica que o processo de feedback nesta organização foi um fator negativo na implantação do BSC.

- Organização interna para a implantação do BSC

Em toda empresa, espera-se que na concretização de uma mudança, seja necessário uma organização interna, de maneira a prepará-la para receber a mudança. Em relação a esse quesito, 90,5\% dos participantes acreditam seja importante ou muito importante; ao mesmo tempo em que, apenas $9,5 \%$ afirmam concordar totalmente que a empresa, na prática, tenha se organizado internamente para a implantação do BSC. Logo, esse se colocou como um fator restritivo na Porto Freire Engenharia.

- Tomada de decisões em tempo hábil

A literatura apresenta diversos casos de empresas que fracassaram na implantação de um BSC, e um dos pontos mais citados é a ausência de tomada de decisões em tempo hábil. Acredita-se seja muito importante que a empresa esteja sempre pronta a tomar todas as decisões no momento certo. Na pesquisa, a maioria dos participantes $(95,2 \%)$ percebe a importância de se priorizar as decisões, considerando esse fator como importante ou muito importante. Contudo, apenas 9,5\% afirmaram estar totalmente em acordo com a ocorrência da tomada de decisões em tempo hábil na empresa. Logo, este foi um fator restritivo.

- Suportes técnicos de consultorias

Bessa (2005) identificou a presença de consultorias na implantação do BSC no SENAI como um fator potencializador, de maneira que esse suporte pode fornecer todo o aparato teórico e técnico que a organização necessitava para realizar a implementação da ferramenta corretamente. Kaplan e Norton (1997) também acreditavam que o processo tendia a ser mais efetivo quando a organização contava com esse tipo de suporte para o desenvolvimento e implantação do BSC. Os resultados apontaram para uma unanimidade em relação à importância 
Roberta Catrib de Azevedo Lemos - Maria Naiula Monteiro Pessoa - Sandra Maia dos Santos - Augusto Cézar de Aquino Cabrak Debora Cardoso do Nascimento

dos suportes técnicos no processo de implantação do BSC; quanto à utilização desses suportes durante o processo na Porto Freire Engenharia, aproximadamente $81 \%$ concordaram totalmente que a empresa tenha feito uso desses. Constata-se que as consultorias foram fatores realmente potencializadores à implantação do BSC nesta empresa, tanto em relação à percepção de sua importância pelos funcionários, quanto pela sua presença durante o processo.

- Presença do pensamento estratégico

No entendimento de Kaplan e Norton (2000), a manutenção do pensamento estratégico é essencial na implantação do BSC, pois é através do pensamento estratégico que a organização é capaz de antever situações e solucionar problemas. Dos participantes, 100\% acreditam que seja importante ou muito importante a presença do pensamento estratégico para o BSC. Ao mesmo tempo, constatou-se que apenas $47,6 \%$ afirmaram estar totalmente de acordo quanto à existência do pensamento estratégico na Porto Freire Engenharia quando da implantação do BSC. Enquadrando-se, portanto, esse quesito como restritivo.

- Resolução dos conflitos de interesses

Cavalcante Neto (2004) estudou duas empresas e constatou que numa delas não houve qualquer barreira quanto aos conflitos de interesses e na outra houve sim diversos conflitos de interesses, mas que foram considerados como barreiras suplantadas. Na implantação de um BSC é importante que não haja conflitos de interesses, ou que estes sejam suplantados quanto existirem. No âmbito da empresa estudada, $85,6 \%$ dos respondentes consideraram esse fator como importante ou muito importante num processo de implantação do BSC. Entretanto, apenas $14,1 \%$ estavam totalmente de acordo que os conflitos de interesses existente à época da implantação do Balanced Scorecard tenham sido solucionados a tempo. Logo, esse fator se apresentou como restritivo à implantação do BSC nesta organização.

- Disponibilização de recursos necessários à implantação

Na visão de Kaplan e Norton (1997), a menos que recursos concretos sejam direcionados para a realização dos objetivos estratégicos, eles serão apenas metas distantes, e não alvos tangíveis capazes de assegurar comprometimento. A totalidade dos participantes considerou como importante ou muito importante a disponibilização dos recursos necessários à implantação do BSC; ao mesmo tempo em que apenas $38,1 \%$ concordaram que a disponibilidade de recursos 
Roberta Catrib de Azevedo Lemos - Maria Naiula Monteiro Pessoa - Sandra Maia dos Santos - Augusto Cézar de Aquino Cabrak Debora Cardoso do Nascimento

na Porto Freire Engenharia ocorreu, exatamente, de acordo com a necessidade do processo. Com isso, percebe-se que os colaboradores possuem a consciência da importância desse fator, mas não concordaram que esse recurso tenha sido disponibilizado sempre que necessário, de maneira que esse fator foi restritivo à implantação do BSC.

- Consideração dos aspectos culturais no processo

Campos (1998) afirma que uma mudança cultural pode ser mais difícil do que qualquer outra mudança, mas a capacidade de comunicação efetiva de informação pode tornar esse processo menos complicado. Dos participantes da pesquisa, 95,2\% avaliaram como importante ou muito importante a consideração dos aspectos culturais para a implantação do BSC. Ao mesmo tempo, apenas $28,6 \%$ estão totalmente em acordo com a afirmação de que os aspectos culturais tenham sido considerados. Logo, este foi considerado um fator restritivo.

- Balanceamento das quatro perspectivas do BSC

Kaplan e Norton (1997) reforçam a necessidade de a implantação do BSC estar focada no balanceamento das quatro perspectivas. Os participantes da pesquisa perceberam a importância dessa questão na implantação de um scorecard, dado que 100\% responderam considerar esse balanceamento como importante ou muito importante. Em relação à implantação na Porto Freire, 66,6\% estão totalmente de acordo que as perspectivas forambalanceadas. Portanto, esse fator foi considerado como potencializador.

- Preservação da identidade corporativa

A identidade da organização precisa ser preservada em todas as fases do processo estratégico, no desenvolvimento da estratégia, na implantação da estratégia ou modelo de gestão estratégica e ainda no gerenciamento da estratégia após a implantação do BSC (KAPLAN E NORTON, 2007). Dos respondentes, 95,2\% consideraram a preservação da identidade corporativa como importante ou muito importante na implantação de um BSC. Ao mesmo tempo, 52,4\% afirmaram estar totalmente de acordo que a identidade da Porto Freire tenha sido preservada durante. Logo, essa questão foi considerada como fator potencializador.

- Comportamento ativo dos envolvidos

Na implantação do BSC, é importante que os envolvidos estejam empenhados e se comportem ativamente. Na empresa, $85,7 \%$ dos participantes afirmam que essa questão é muito 
Roberta Catrib de Azevedo Lemos - Maria Naiula Monteiro Pessoa - Sandra Maia dos Santos - Augusto Cézar de Aquino Cabrak Debora Cardoso do Nascimento

importante para que a implantação possa ocorrer corretamente. Entretanto, constatou-se que apenas $19 \%$ desses respondentes estão totalmente em acordo de que houve um comportamento ativo dos envolvidos. É interessante analisar esse ponto, pois os respondentes, embora tenham participado da implantação do BSC, e reconheçam a importância de manter um comportamento ativo, acreditam que este comportamento não predominou ao longo do processo. Portanto, esse também foi um ponto restritivo na implantação do BSC.

- Obediência ao cronograma estabelecido

De acordo com os resultados, $90,4 \%$ consideram o cumprimento do cronograma estabelecido como sendo um ponto importante ou muito importante. Do total de participantes, $5 \%$ deixaram de responder a essa questão; 32,9\% estavam muito ou parcialmente em desacordo em relação à obediência ao cronograma estabelecido na Porto Freire Engenharia; enquanto 52,3\% estavam parcialmente em acordo quanto a essa questão. Percebeu-se que esse foi um dos fatores mais negativos no processo de implantação do BSC nesta empresa.

O Quadro 4 apresenta um resumo dos quesitos analisados, destacando os principais fatores potencializadores e restritivos à implantação do BSC na Porto Freire Engenharia.

Quadro 4: Resumo dos resultados da pesquisa.

\begin{tabular}{|c|c|}
\hline FATORES POTENCIALIZADORES & FATORES RESTRITIVOS \\
\hline Suporte técnico de consultorias & Envolvimento da alta direção \\
\hline $\begin{array}{l}\text { Balanceamento das quatro perspectivas do } \\
\text { BSC }\end{array}$ & Comprometimento dos colaboradores \\
\hline Preservação da identidade corporativa & Identificação prévia das mudanças necessárias \\
\hline |---------------------- & Comunicação do processo em todas as etapas \\
\hline & Incentivos para motivação dos colaboradores \\
\hline ---------------------- & Promoção de diálogos transparentes \\
\hline --------------------- & Escolha de estratégia compatível com objetivos \\
\hline ---------------------- & Mapeamento dos processos \\
\hline ---------------------- & $\begin{array}{l}\begin{array}{l}\text { Fornecimento de feedback nas fases do } \\
\text { processo }\end{array} \\
\end{array}$ \\
\hline ---------------------- & Organização interna \\
\hline ---------------------- & Tomadas de decisão em tempo hábil \\
\hline ---------------------- & Pensamento estratégico \\
\hline --------------------- & Resolução de conflitos de interesse \\
\hline --------------------- & Disponibilização de recursos \\
\hline ---------------------- & Consideração dos aspectos culturais \\
\hline --------------------- & Comportamento ativo dos envolvidos \\
\hline --------------------- & Obediência ao cronograma estabelecido \\
\hline
\end{tabular}

Fonte: elaborado pelos autores (2010). 
Roberta Catrib de Azevedo Lemos - Maria Naiula Monteiro Pessoa - Sandra Maia dos Santos - Augusto Cézar de Aquino Cabrak Debora Cardoso do Nascimento

\subsubsection{Características positivas e negativas da empresa}

Nesta seção, são apresentados os dados referentes ao terceiro bloco do questionário, em que se busca identificar a relevância atribuída às características da organização no processo de implantação do BSC.

Tabela 1 - Percepção dos colaboradores quanto às características da empresa.

\begin{tabular}{l|l|l|l}
\hline Características da Empresa & Média & Características da Empresa & Média \\
\hline porte da empresa & 5,48 & estrutura hierárquica & 5,19 \\
\hline número de colaboradores & 5,19 & descentralização da informação & 5,00 \\
\hline modelo de gestão & 5,24 & ocorrência de mudanças & 5,00 \\
\hline ramo de negócio & 5,48 & nível de planejamento & 5,43 \\
\hline nível escolaridade & 5,48 & nível de organização & 5,43 \\
\hline mecanismo de informação & 5,14 & experiência no ramo & 5,19 \\
\hline sistema de informatização & 5,05 & capacidade de inovação & 5,76 \\
\hline disponibilidade financeira & 5,29 & volume de obras & 5,19 \\
\hline conhecimento da ferramenta & 5,29 & -------------- & -------- \\
\hline
\end{tabular}

Fonte: elaborado pelos autores (2010).

Todas as características apresentaram uma média próxima de cinco pontos, sendo, portanto, consideradas potencializadoras. A capacidade de inovação apresentou uma maior pontuação, média de 5,76, indicando que os participantes julgaram que uma organização que apresenta uma postura inovadora possui melhores chances de alcançar os resultados pretendidos com a implantação do BSC e, com isso, da execução da estratégia. Ademais, o porte da empresa, o ramo do negócio e o nível de escolaridade obtiveram a segunda maior média, 5,43, evidenciando a relevância atribuídas a estas características específicas do negócio e dos colaboradores. O nível de planejamento e nível de organização obtiveram a terceira maior média, 5,43, sendo considerados pelos colaboradores como facilitadores do processo. As demais características obtiveram médias próximas de cinco pontos.

\subsection{A VISÃO DA ALTA DIREÇÃO EM RELAÇÃO AOS FATORES CARACTERÍSTICOS DA IMPLANTAÇÃO DO BSC}

Este segmento apresenta os resultados referentes aos dados oriundos das entrevistas, conforme as categorias de análise apresentadas na metodologia. 
Roberta Catrib de Azevedo Lemos - Maria Naiula Monteiro Pessoa - Sandra Maia dos Santos - Augusto Cézar de Aquino Cabrak Debora Cardoso do Nascimento

- Dependência do planejamento para o sucesso da execução da estratégia

Os participantes foram unânimes ao ressaltar a importância que a empresa atribui ao planejamento estratégico, que foi desenvolvido de maneira natural, devido a uma cultura consolidada na empresa. Kaplan e Norton (2000) afirmam que a execução da estratégia é mais importante que a qualidade da estratégia em si, mas não se pode executar aquilo que não está definido e é através do planejamento estratégico que a estratégia é consolidada. A compreensão clara da alta direção no que se refere à facilidade com que o planejamento estratégico foi desenvolvido na organização está respaldada na prática de planejamento já existente na empresa desde a sua concepção, como evidenciado a seguir:

Na verdade, o planejamento estratégico nesta empresa aconteceu de maneira natural, pois como sempre vendíamos planejamento, tínhamos que vivenciar isso para que pudéssemos vender de maneira mais eficiente, tínhamos que ter isso intrínseco na nossa forma de atuação (ENTREVISTADO 1).

A gente sempre trabalhou com planejamento e planejamento estratégico. Mesmo não sendo formalmente, a gente trabalhava com esse foco, pois nos planejávamos para o futuro, isso era constante na empresa (ENTREVISTADO 3).

A empresa utilizou-se do planejamento como uma ferramenta que gerou vantagem competitiva, ao identificar um nicho em que poderia atuar de forma diferenciada. Desenvolveu um sistema de financiamento próprio, por meio do qual a obra era financiada com o dinheiro dos consumidores, que, por sua vez, formavam grupos de condomínio que poderiam adquirir seu imóvel por meio de compra programada, a preço de custo, tendo apenas que fiscalizar a empresa responsável. Esta visão é evidenciada a seguir:

Quando nós quisemos começar esse trabalho, havia uma escassez de recurso no sistema financeiro e a gente vislumbrou um nicho, um espaço, que poderia ser ocupado por um sistema alternativo. Então, a gente criou esse sistema de construção a preço de custo, fazendo com que as pessoas se planejassem e as obras pudessem ser realizadas de maneira autofinanciada (ENTREVISTADO 2).

Desenvolver um planejamento estratégico sem vivenciar na organização práticas de planejamentos, pode comprometer todo o processo, na visão dos participantes deste estudo.

- Objetivação da estratégia da organização 
Roberta Catrib de Azevedo Lemos - Maria Naiula Monteiro Pessoa - Sandra Maia dos Santos - Augusto Cézar de Aquino Cabrak Debora Cardoso do Nascimento

A principal motivação apontada quanto ao uso do BSC foi a possibilidade de mensuração da execução da estratégia. Kaplan e Norton (1997) demonstraram essa característica quando criaram esse modelo com foco em indicadores de desempenho que procuram objetivar a análise dos objetivos estratégicos de cada perspectiva. Os depoimentos abaixo demonstram a necessidade sentida em mensurar resultados, especialmente por ser uma empresa de engenharia, como ilustrado a seguir:

Sete ou oito anos atrás, falava-se muito de visão de futuro e, como somos uma empresa de engenharia, sentíamos uma grande necessidade de mensuração. Com isso, o Balanced Scorecard apresentou-se como um modelo ideal, pois se propõe exatamente a mensurar objetivos (ENTREVISTADO 1).

O Balanced Scorecard foi a ferramenta que mais aderiu a nossa cultura organizacional, pois a gente consegue enxergar melhor a estratégia da empresa e colocá-la em prática, através da mensuração (ENTREVISTADO, 3).

- Aparato teórico e técnico da consultoria na implantação do BSC

A visão objetiva apresentada por essa assessoria permitiu que a organização pudesse aliar as características teóricas do BSC às características próprias do negócio, mantendo a sua essência. Além disso, contribuiu para a manutenção das relações de causa e efeito entre as medidas de resultado e os vetores de desempenho, como evidenciado a seguir:

A consultoria externa foi extremamente importante e nós não teríamos conseguido sem eles, pois a gente tende a querer moldar a ferramenta a nossa visão, o que é um erro, pois é necessário que não se esqueça as relações de causa e efeito. Muitas vezes a gente tentava fugir dessas relações e o moderador sempre nos trazia de volta a base teórica que fundamenta a ferramenta (ENTREVISTADO 1).

Inicialmente fizemos o planejamento estratégico, considerando análise SWOT e, com isso, traçamos uma estratégia. Depois nós contratamos uma assessoria para implantar o BSC. Essa consultoria nos deu o aparato teórico. Fomos reformulando nossa estratégia e agora vimos que precisávamos ajustar a estrutura e contar com uma assessoria interna para gerir esse processo (ENTREVISTADO 2).

Além de uma consultoria externa, a empresa está implementando uma assessoria interna, que tem por objetivo incentivar a utilização do BSC como um instrumento de gestão. 
Roberta Catrib de Azevedo Lemos - Maria Naiula Monteiro Pessoa - Sandra Maia dos Santos - Augusto Cézar de Aquino Cabrak Debora Cardoso do Nascimento

- Sensibilização dos envolvidos para a utilização do BSC como instrumento de gestão

A alta direção demonstrou envolvimento durante o processo de sensibilização, como defendem Kaplan e Norton (1997). Conforme os entrevistados, o processo ainda está no início, tendo a empresa utilizado diversas estratégias para alcançar os envolvidos, tais como a exposição do mapa estratégico em murais e informativos enviados por correio eletrônico.

A sensibilização dos colaboradores é uma grande dificuldade para a implantação de qualquer ferramenta e, até hoje, temos esse tipo de problema. No início, optamos por desenvolver a ferramenta na empresa através de um grande grupo, contando com a participação de diversas funções dentro da organização, pois intuíamos que assim envolveríamos mais as pessoas. No entanto, isso não aconteceu, pois o grupo era composto de pessoas com visões muito diferentes (ENTREVISTADO 1).

Ainda estamos nesse processo. É preciso ter uma gestão com foco nos indicadores e acredito que estamos caminhando para isso, pois não adianta termos um BSC para dizer que temos, ele precisa ser efetivo. Antes a gente envolvia um grupo maior de pessoas e tivemos um problema relacionado ao fato de que tínhamos problemas em relação à definição dos responsáveis, de maneira que muitas vezes as reuniões tornavam-se contemplativas, expositivas, mas não gerenciais (ENTREVISTADO 2).

Agente descobriu uma maneira de envolver as principais pessoas da organização e fornecer a elas o acesso ao BSC. Como nós fazíamos antes, lotando auditórios, não era muito interessante e a reunião tornava-se muito contemplativa. Com o grupo menor fica mais dinâmico e torna-se mais executivo (ENTREVISTADO 3).

Apesar de os relatos demonstrarem que a empresa tem alcançado melhores resultados quanto à sensibilização dos colaboradores e a utilização da ferramenta, a alta direção possui plena convicção de que precisa estar mais envolvida no processo, para que consiga gerar o devido comprometimento com a estratégia nos demais constituintes da empresa.

- Construção de cultura organizacional direcionada ao trabalho disciplinar

A cultura da empresa foi considerada positiva para a implantação do scorecard. Além disso, foi citado que o principal fator que viabilizou essa aderência da cultura ao BSC foi o fato da empresa estar focada na busca da sua identidade, o que, para alguns dirigentes, mantém a organização focada, fortalecendo a estratégia planejada, conforme relato a seguir: 
Roberta Catrib de Azevedo Lemos - Maria Naiula Monteiro Pessoa - Sandra Maia dos Santos - Augusto Cézar de Aquino Cabrak Debora Cardoso do Nascimento

A nossa cultura é totalmente positiva a esse processo. Como já foi dito, a gente vendeu planejamento, de maneira que sempre tivemos o planejamento implícito na nossa forma de atuação. O cerne da nossa missão está relacionado à viabilização da prosperidade das pessoas e, para nós, essa prosperidade é alcançada através do planejamento (ENTREVISTADO 1).

A cultura da empresa é positiva a implantação de uma ferramenta como essa, pois trabalhamos com muito foco na nossa identidade e acredito que a estratégia está mais clara, porque a identidade está muito clara. Com uma identidade clara, o Balanced Scorecard ou qualquer outra ferramenta é implantada mais facilmente, pois o Balanced Scorecard é apenas uma ferramenta para gerir a estratégia que está fundamentada na idéia central da organização (ENTREVISTADO 3).

Acredita-se que o estágio em que a organização encontra-se é de maturação, de maneira que a cultura organizacional precisa obter um maior desenvolvimento para ser capaz de incentivar a aderência desejada ao BSC pela alta direção da empresa.

- Perspectiva de alcance dos resultados esperados

Os dirigentes acreditam que o resultado esperado ainda não foi alcançado, mas que a empresa está evoluindo de maneira satisfatória. Os entrevistados apresentam uma postura muito segura quanto ao alcance do sucesso esperado pela implantação dessa ferramenta.

Dizer que alcançamos o sucesso esperado é muita pretensão, pois acredito que ainda estamos no meio do processo, mas temos convicção que essa ferramenta é muito importante e que vamos conseguir o resultado esperado (ENTREVISTADO 2).

Já evoluímos muito, mas ainda falta evoluirmos. Ainda precisamos focar mais numa administração visual da nossa estratégia. No entanto, acredito que estamos em um estágio de intermediário a alto em relação à maioria das empresas, mas ainda falta chegarmos no ponto em que o Balanced Scorecard incentiva o modelo de gestão. Acho que em termos de objetivos financeiros, estamos bem evoluídos, mas ainda precisamos desenvolver melhor as demais perspectivas (ENTREVISTADO 1).

A organização apresenta os objetivos relacionados à perspectiva financeira mais evoluídos, de maneira que os demais ainda estão em fase de desenvolvimento. Os pontos fracos do scorecard da Porto Freire foram vistos como reflexos de erros do passado, pois se afirmou que 
Roberta Catrib de Azevedo Lemos - Maria Naiula Monteiro Pessoa - Sandra Maia dos Santos - Augusto Cézar de Aquino Cabrak Debora Cardoso do Nascimento

o BSC é apenas um espelho da organização, que precisa sempre focar na qualidade da estratégia, para que possa ocorrer a evolução da ferramenta.

Com a análise apresentada, foram identificados alguns fatores potencializadores e restritivos à implantação do BSC, como também algumas especificidades inerentes à Porto Freire Engenharia. As práticas de planejamento, as consultorias e a mensuração da estratégia foram apontadas como fatores potencializadores. No entanto, também foram identificados alguns fatores restritivos. Ainda que parcialmente, a cultura organizacional foi vista como um deles, por não ser direcionada a um trabalho mais disciplinar e orientado, essencial no modelo do scorecard. Também foi citada a ineficiência do processo de sensibilização dos colaborares, que está sendo revisto pelos dirigentes da empresa, no intuito de apresentar um maior envolvimento da alta direção, de maneira que estimule o comprometimento dos demais. Por fim, a perspectiva e alcance dos resultados foi considerada fator restritivo.

\section{CONCLUSÃO}

Este estudo se propôs a identificar os principais fatores potencializadores e restritivos à implantação do Balanced Scorecard em uma empresa de construção civil, no intuito de que outras organizações não incorram nos mesmos erros e se orientem pelos acertos. Nesse sentido,além de uma pesquisa documental, foi realizada pesquisa de campo mediante um questionário respondido pelos colaboradores que já eram integrantes da Porto Freire engenharia no momento em que o Balanced Scorecard foi implantado. Foi, também, utilizado um roteiro de entrevista, aplicado a todos os componentes da alta direção.

Em relação à pesquisa documental, considerando-se os relatórios gerencias do período de 2003 a 2009, constatou-se que a empresa realizou o processo necessário para a implantação do BSC para a gestão de sua estratégia, pois partiu de uma análise SWOT e evoluiu com o planejamento estratégico.

Quanto aos dados do questionário, os resultados indicam que, do conjunto dos vinte fatores considerados, apenas três foram apontados como potencializadores à implantação do 
Roberta Catrib de Azevedo Lemos - Maria Naiula Monteiro Pessoa - Sandra Maia dos Santos - Augusto Cézar de Aquino Cabrak Debora Cardoso do Nascimento

Balance Scorecard na Porto Freire Engenharia: suporte técnico de consultorias, balanceamento das quatro perspectivas e preservação da identidade corporativa. Importante ressaltar que a percepção dos entrevistados em relação ao grau de importância dos quesitos analisados diferencia-se do grau de realização percebida na implementação do BSC na empresa pesquisada. Para os respondentes, todos os vinte pontos selecionados enquadram-se como importantes ou muito importantes para a implantação de um BSC.

Em relação aos dados das entrevistas, as práticas de planejamento, as consultorias e a mensuração da estratégia foram apontadas como fatores potencializadores. A cultura organizacional, o processo de sensibilização dos colaborares e a perspectiva e alcance dos resultados foram considerados fatores restritivos.

Esta pesquisa não é conclusiva, mas abre caminhos para reflexões e para que outros estudos sejam realizados na busca de um processo de implantação de um Balanced Scorecard mais participativo e planejado.Espera-se que as constatações evidenciadas possam subsidiar as discussões sobre o processo de desenvolvimento e implantação do Balanced Scorecard nas empresas, bem como suscitar outras investigações no campo.

\section{POTENTIALIZING AND RESTRICTIVE FACTORS IN THE IMPLEMENTATION OF THE BALANCED SCORECARD: A STUDY IN A CONSTRUCTION FIRM IN THE STATE OF CEARÁ}

\section{ABSTRACT}

The Balanced Scorecard (BSC), better known as a method to translate a firm's mission and strategy into a broad set of performance measurements, has been used for the strategic management of organizations, serving as a measurement system that, besides the financial goals, includes performance characteristics. This research, which was carried out through a case study, aims at identifying the main potentializing and restrictive factors in the implementation of the BSC in a 
Roberta Catrib de Azevedo Lemos - Maria Naiula Monteiro Pessoa - Sandra Maia dos Santos - Augusto Cézar de Aquino Cabrak Debora Cardoso do Nascimento

civil construction firm in the state of Ceará, the Porto Freire Engineering. The bibliographical research, which was based on the managerial reports of the 2003 to 2009 period, showed that the firm conducted the process necessary to the implementation of the BSC for the management of its strategy. The field data were collected through questionnaires and interviews applied, respectively, to the employees and managers who already were in the firm when the BSC was implemented. The questionnaire data show that, among the set of twenty factors considered in the research, only three were deemed potentializing in the implementation of the BSC: technical support, balancing of the four perspectives and preservation of the corporate identity. The interview data show that the planning practices, the consultancy services and the strategy measurement were deemed potentializing factors. The organizational culture, the sensitization process of the employees and the perspective and reach of the results were deemed restrictive factors.

Key-words: Strategy. Balanced scorecard. Civil construction.

\section{REFERÊNCIAS}

BARDIN, L. Análise de conteúdo. 5. ed. Lisboa: Edições 70, 2008.

BESSA, F. E. H. Implantação de indicadores balanceados em instituições sem fins lucrativos: um estudo de caso com o Balanced Scorecard no SENAI-CE. Dissertação (Mestrado Profissional em Controladoria). Programa de Pós-Graduação da Universidade Federal do Ceará, UFC, Fortaleza, 2005.

BRANDÃO, H. P. et al. Gestão de desempenho por competências: integrando a gestão por competências, o Balanced Scorecard e a avaliação 360 graus. Revista de Administração Pública, v. 42, n. 5, p. $75-98$, set./out., 2008.

BRASIL. Lei no 10.165, de 27 de dezembro de 2000. Altera a legislação sobre política nacional do meio ambiente. Diário Oficial [da] República Federativa do Brasil, Brasília, DF, 27 dez. 2000. Disponível em: <http://www.planalto.gov.br>. Acesso em: 24 de novembro de 2009.

Cavalcante Neto (2004) 
FATORES POTENCIALIZADORES E RESTRITIVOS À IMPLANTAÇÃO DO BALANCED SCORECARD: UM ESTUDO EM UMA EMPRESA DE CONSTRUÇÃO CIVIL DO CEARÁ

Roberta Catrib de Azevedo Lemos - Maria Naiula Monteiro Pessoa - Sandra Maia dos Santos - Augusto Cézar de Aquino Cabrak Debora Cardoso do Nascimento

CAMPOS, J. A. Cenário balanceado: painel de indicadores para a gestão estratégica dos negócios. São Paulo: Aquariana, 1998.

GALAS, E. S.; PONTE, V. M. R. O Balanced Scorecard e o alinhamento organizacional: um estudo de casos múltiplos. In: Encontro do ANPAD, 29, 2005, Brasília. Anais...Rio de Janeiro: ANPAD, 2005. 1 CD ROM.

HERRERO FILHO, E. Balanced scorecard e a gestão estratégica. 10. ed. Rio de Janeiro: Elsevier, 2005.

KAPLAN, Robert S.; NORTON, David P. The balanced scorecard: measures that drive performance. Harvard Business Review, jan./fev., p. 71-79, 1992.

A estratégia em ação: balanced scorecard. Rio de Janeiro: Campus, 1997.

Organização orientada para a estratégia: como as empresas que adotam o balanced scorecard prosperam no novo ambiente de negócios. Rio de Janeiro: Campus, 2000.

.Kaplan e Norton na prática. 6. ed. Rio de Janeiro: Elsevier, 2004.

McKENZIE, F. C.; SHILLING, M. D. Avoiding Performance Neasuyrement Traps:Ensuring Effective Incentive Design and Implementation. Compensation\&BenefitsReview, v. 30, p. 57-65,1998.

OLIVEIRA, M.; FREITAS, H. Seleção de indicadores para tomada de decisão: a percepção dos principais intervenientes na construção civil. ReAd, Rio Grande do Sul, Edição especial impressa, 24, vol. 7, n. 6, dez., 2001, p. 175 - 178. Disponível em: <http://www.read.adm.ufrgs.br>. Acesso em: 03 de julho de 2009.

OLVE, G.; ROY, J.; WETTER, M. Condutores da performance: um guia prático para o uso do balancedscorecard. Rio de Janeiro: Qualitymark, 2001.

PORTO FREIRE. Identidade organizacional. Disponível em <www.portofreire.com.br>. Acessado em 15 de janeiro de 2010.

PRADO, L. Dez riscos na implantação do Balanced Scorecard. Disponível em: <http://www.lauroprado.tripod.com.>. Acesso em: 18 de agosto de 2009.

PRIETO, V. C. et al. Fatores críticos na implementação do Balanced Scorecard. Gestão e Produção, São Carlos, v. 13, n. 1, jan./abr., p. 81-92, 2006.

YIN, R. K. Estudo de caso: planejamento e método. 3. ed. Porto Alegre: Bookman, 2005. 